\title{
On Intrinsic Rotation of Bodies
}

\section{Ogaba Philip Obande}

Retired, Department of Chemistry, Ahmadu Bello University, Zaria, Nigeria

Email: gababands@gmail.com

How to cite this paper: Obande, O.P. (2019) On Intrinsic Rotation of Bodies. Journal of High Energy Physics, Gravitation and Cosmology, 5, 868-883. https://doi.org/10.4236/jhepgc.2019.53043

Received: May 5, 2019

Accepted: July 12, 2019

Published: July 15, 2019

Copyright $\odot 2019$ by author(s) and Scientific Research Publishing Inc. This work is licensed under the Creative Commons Attribution International License (CC BY 4.0).

http://creativecommons.org/licenses/by/4.0/

\begin{abstract}
Spontaneous rotation of matter is investigated. The results provide further evidence in support of earlier indications of a wave-only reality in which the quantum energy packet exists in the form of a conjugate wave-particle doublet. It reveals that matter, be it wave or particle, is a harmonic oscillator defined with full spectrum of the usual mechanical properties of simple harmonic motion SHM. Notably, the quantum energy packet's stress field correlates with radius to generate intrinsic torque, it motivates spontaneous rotation at all levels of the cosmic mass scale from the electron to the universe; its atomic and natural units are $\Gamma_{a u(w)}=3.162 \times 10^{-25} \mathrm{~kg} \cdot \mathrm{m}^{3} \cdot \mathrm{s}^{-2}$ and $\Gamma_{a u(p)}=3.867 \times 10^{-47} \mathrm{~kg} \cdot \mathrm{m}^{3} \cdot \mathrm{s}^{-2}$ respectively for matter's wave (bosonic) and particulate (fermionic) forms. The proton's observational internal pressure $\sigma_{\text {proton }} \sim 10^{35} \mathrm{~Pa}$ reported recently by Burkert et al. deviates markedly from the theoretical value $\sim 10^{22} \mathrm{~Pa}$, the difference attributes to challenges with existing energy measurement procedures. Velocities of electron waveform in random thermal motion evaluated with the new approach agree remarkably well with values obtained with kinetic-molecular theory KT; much more importantly, the analysis reveals existence, at standard conditions, of electron waveform's hyper-luminal root-mean-square velocity, $v_{r m s(e w)}=1.004 \times 10^{27} \mathrm{~m} \cdot \mathrm{s}^{-1}$, if verified, this finding might inform on-going neutrino research. The evidence suggests that effects formalized in the theories of thermodynamics and kinetics trace to mobile torque fields.
\end{abstract}

\section{Keywords}

Atomic Internal Pressure, Atomic Mechanical Property, Atomic Torque Unit, Hyper-Luminal Speed, Metric Space Expansion, Spontaneous Rotation

\section{Introduction}

As observed recently, Ellis \& Silk [1], physics is, indeed, "Faced with difficulties 
in applying fundamental theories to the observed universe...”. Of immediate relevance is the search for tenable account of scale-free observational rotation of: the photon, Beth [2], Leach et al. [3]; the atom, Jaffe [4], Peterson [5]; the galaxy, Raghuprasad [6], Tsati et al. [7] and the active galactic nucleus (black hole), McClintock et al. [8], Imanishi et al. [9]; they all rotate but the causality remains unknown, Williams and van der Mark [10]. The prevailing notion is undecided. At the atomic level, we have brilliant cases that attempt to convince that choreographed dance steps of constituent "embryonic" quarks and gluons inside the atomic sac give rise to proton spin with the gluon field dominating the stage, Shea [11]. To our best knowledge, no attempt exists to extend the idea to suband supra-proton species with explanation for how well the notion sits with the atomic point-mass concept. At the cosmic level the authorities attribute rotation to imbalance ("asymmetry") of forces acting on a body in gravity-motivated collapse, Spagna [12], Palacios [13], Benesch [14], Crawford [15]. Many, particularly among the student population, find this explanation less than satisfactory as evident from lively exchanges at the Physics Forum [16] which center on the popular but erroneous notion that “The spin of elementary particles [isn't] 'normal' Newtonian force vector but rather a property of the particle itself...".

The privilege of an observational theory of the atom now enables us to see that Dirac [17] was in error in his concepts of the "singularity" and the atomic "point-mass". As proposed earlier by Born [18], the atom is indeed "extended" and imbued with elaborate structures and mechanical properties, Golubev [19], Obande [20]. Sadly, Dirac [17] also established the supremacy of elegance with the historic position that the aim "is not so much to get a model of an electron as to get a simple scheme of equations which can be used to calculate all the results that can be obtained from experiment"; the position sustains a storm that rages on, Hossenfelder [21], Wilczek [22]. Theory rules out the "singularity" and rejects the atomic "point mass" conjecture, we side with others, Rugh \& Zinkernagel [23] who attribute the current crisis to these two foundational errors. The positions are contrary to nature, therefore, cannot describe same; they leave no room for contemplation of structured co-ordinate space and intrinsic mechanical properties of the quantum energy packet. Perpetual motion of any kind cannot exist without a perpetuating agent and the latter is inconceivable for a point mass and structureless space.

No one seems to question the basis of Newton's translational second law, not so with the rotational second law, physicists are interested in how perpetual rotation is contrived. Of course, a body cannot be in perpetual rotational motion unless impacted upon by a perpetuating torque, see, for instance, Rees [24] but, no one seems to know just how nature manufactures torque in an isolated body. The result of a recent attempt to trace the physical origin of torque and of the rotational second law was not quite satisfactory, Cross [25], neither was a subsequent attempt to improve upon the first, Gorbatyy and Tarsov [26]. In any case, use of a force carrier in the form of a rod connecting the mass to a pivot automatically rules out any semblance of Cross' model to an isolated body such 
as the atom or the galaxy. The problem centers on a simple question: How does nature, using no force carrier, create matter's rotation at each rung of the hierarchical structures that define the cosmic mass scale? We have accumulated overwhelming evidences in support of the "extended" atom, see, e.g., Obande ([20] [27]); based on the atom's internal structure and dynamics, we attempt here a quantitative account of Newton's second law of translational, rotational or any other mode of motion.

\section{Wave, Particle or Wave-Particle?}

The outcome of a debate on the nature of reality: Is it field or particle? SCIAM [28], expertly summarized by Kuhlman [29], came up with the unavoidable conclusion that reality is wave-only. It tallies with consistent theoretical and experimental results reported by investigators widely separated in time and in space, Descartes [30], Madelung [31], Born [18], Macken [32], Hobson [33], Obande [34], Consiglio [35], Kirakosyan [36]; it now seems capable of generating renewed research interest, Wilczek [37], Colbert and Renner [38]. As observed recently, "whether it appears as radiation or as mass, energy is a radiative phenomenon", Laidlaw [39]. Musser [40] suggests that "Physicists will need to find some new foundational structure ...", we beg to differ, there has, for over four centuries, always been one-the wave-only imponderable material vacuum field defined with articulated structures and mechanical properties. One of the most informative descriptions of this "foundational structure" is available at Physics Forum posted by a well-informed source with the pseudonym "Good Elf" [41] sadly, it constrains independent interaction. Consistent results of our investigations point to a reality that is essentially field defined, on the one hand, with the following fundamental quantum vacuum (boson field) characteristics: oscillation frequency $\vartheta_{w}=1.0 \mathrm{~s}^{-1}$, angular speed $c_{o}=2.99792458 \times 10^{8} \mathrm{rad} \cdot \mathrm{s}^{-1}$, not $\mathrm{m} \cdot \mathrm{s}^{-1}$ and energy $E_{w}=h \vartheta_{w}=6.62607 \times 10^{-34} \mathrm{~J}$ and, on the other hand, with the following fundamental quantum matter (fermion field) characteristics:

$\vartheta_{p}=2.034 \mathrm{~s}^{-1}, \quad c^{o}=3.71535229 \times 10^{-14} \mathrm{rad} \cdot \mathrm{s}^{-1}$ and

$E_{p}=h \vartheta_{p}=1.34774 \times 10^{-33} \mathrm{~J}$. It delivers a classical field theory (CFT) absolutely free of existing crises of the probabilistic theory but, it's quantitative approach is easily dismissible for being rather simplistic and desperately devoid of even a pretence at elegance. The approach, however, more than compensates for these "shortcomings" in providing a most formidable theoretical analytical tool, see, for instance, Obande ([20] [42] [43]); here, we employ its analytical power to probe intrinsic rotation of matter.

\section{Procedure}

The quantum packet's field parameters are generated from classical (Newtonian) expressions for radius $r$, density $\rho$, angular speed $\omega$, centripetal force $F$, tensile modulus $\epsilon$, longitudinal stress $\sigma$ and strain $\tau$ using the element's $\vartheta$ and m values. Field-specific correlation of values of these parameters yields the desired prop- 
erty, the details have been reported, Obande ([20] [44] [45]).

\section{Results and Discussion}

The results are presented in two figures and five tables. Figure 1 is a plot of values of the atom's bosonic $r_{w}$ and fermionic $r_{p}$ radii versus mass number $Z_{n}$ while Figure 2 is a plot of $r_{w}$ versus $r_{p}$. Table 1 is a compilation of some of nature's torque fields; Table 2 lists some tensile properties of the atomic harmonic oscillator; Table 3 presents evidence for periodic variation in values of the ratio $r_{w} / r_{p}$; Table 4 is a list of some observational effects of the atom's intrinsic strain and Table 5 presents internal stress (pressure) $\sigma_{p}$ values for a sample of elements in the visible universe $U_{p}^{o}$ and in its invisible analogue $U_{p}^{\prime}$.

The torque field identifies by its unit following dimensional analysis of the coefficient of correlation of two interacting parameters, it must include the generic term Newton-meter $\mathrm{N}$ m. For example, field stress couples with radius to give, $\sigma_{p} r_{p}^{4}=\Gamma_{n u}=3.867 \times 10^{-47} \mathrm{~kg} \cdot \mathrm{m}^{3} \cdot \mathrm{s}^{-2}$, Equation (4), the waveform equivalent is $\sigma_{w} r_{w}^{4}=\Gamma_{a u}=3.162 \times 10^{-25} \mathrm{~kg} \cdot \mathrm{m}^{3} \cdot \mathrm{s}^{-2}$, these coefficients are the primitive causality of rotation, they are the natural unit $\Gamma_{n u}$ and the atomic unit $\Gamma_{a u}$ of torque. Notice the similarity between the difference $8 \times 10^{21}$ and the "molar" constant $N_{A}=6.022 \times 10^{23}$ units. We examine the data in Table 1 .

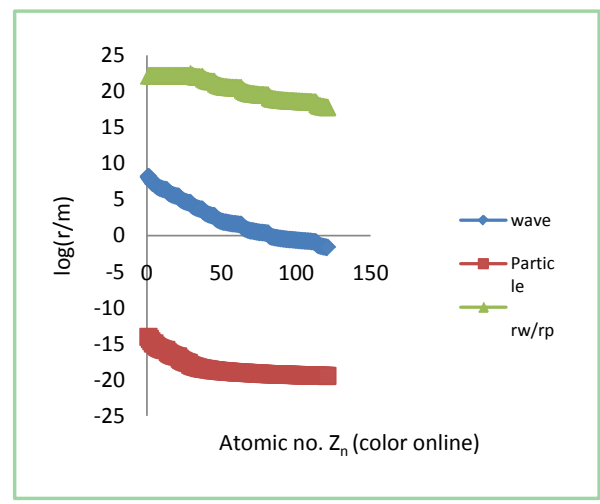

Figure 1. Plot of $\log (r / m)$ vs. $Z_{n}$.

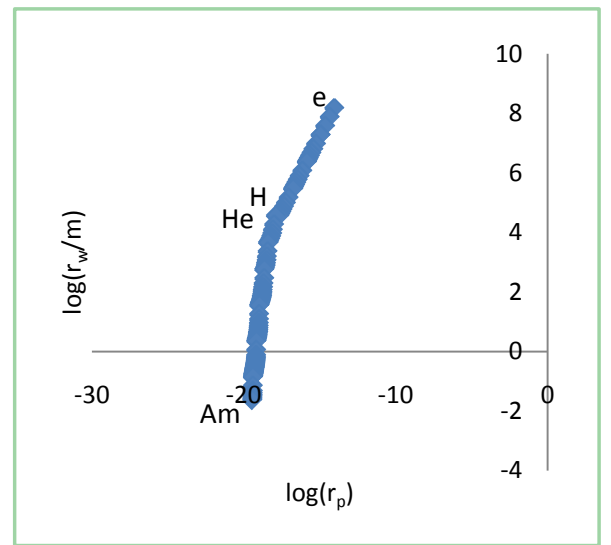

Figure 2. Plot of $\log \left(r_{w} / m\right)$ vs. $\log \left(r_{p} / m\right)$. 
Table 1. Nature's causal torque fields and rotation-motivated effects (ref. Obande [20]).

\begin{tabular}{|c|c|c|c|c|}
\hline Equation & Correlation coefficient & Correlation coefficient & Fundamental & Observational \\
\hline No. & Graphical & Computed & Constant & Effect \\
\hline 1) & $\rho_{p} / \tau_{p}^{4}=2.089 \times 10^{-19}$ & $2.074 \times 10^{-19} \mathrm{~N} \mathrm{~m}$ & $1.602 \times 10^{-19} \mathrm{C}$ & Charge quant._false \\
\hline 2) & $F_{p} / \vartheta_{p}^{2}=5.248 \times 10^{-19}$ & $3.548 \times 10^{-19} \mathrm{~N} \mathrm{~m}$ & $1.602 \times 10^{-19} \mathrm{C}$ & Charge quant._false \\
\hline 3) & $\rho_{w} / \vartheta_{w}^{4}=5.248 \times 10^{-19}$ & $1.069 \times 10^{-19} \mathrm{~N} \mathrm{~m}(\mathrm{~m} / \mathrm{s})^{-3} \mathrm{rad} / \mathrm{s}^{-1}$ & $1.602 \times 10^{-19} \mathrm{C}$ & Charge quant._false \\
\hline 4) & $\sigma_{p} r_{p}^{4}=3.890 \times 10^{-47}$ & $3.867 \times 10^{-47} \mathrm{~kg} \mathrm{~m}^{3} \mathrm{~s}^{-2}$ & Unknown (new) & Rotation of matter \\
\hline 5) & $r_{w} \rho_{w}^{0.25}=2.291 \times 10^{-11}$ & $2.266 \times 10^{-11}(\mathrm{~N} \mathrm{~m})^{0.25}$ & $6.674 \times 10^{-11} \mathrm{~m}^{3} \cdot \mathrm{kg}^{-1} \mathrm{~s}^{-2}$ & Grav. Constant \\
\hline 6) & $\rho_{w} / \epsilon_{w}^{1.33}=2.754 \times 10^{-11}$ & $2.610 \times 10^{-11}\left(\mathrm{mr}^{2} \omega\right)^{-0.3}(\mathrm{~m} / \mathrm{s})^{-2.3}$ & $6.674 \times 10^{-11} \mathrm{~m}^{3} \cdot \mathrm{kg}^{-1} \mathrm{~s}^{-2}$ & Grav. Constant \\
\hline 7) & $r_{p} \rho_{p}^{0.25}=6.792 \times 10^{-6}$ & $5.712 \times 10^{-6}(\mathrm{~N} \mathrm{~m})^{0.25}$ & $\sim 1.0 \times 10^{-6} \mathrm{~m} \cdot \mathrm{s}^{-2}$ (gal.) & Grav. Accl. Const. \\
\hline 8) & $\epsilon_{\mathrm{w}} / \sigma_{w}^{0.75}=2.350 \times 10^{-6}$ & $2.348 \times 10^{-6}(\mathrm{~N} \mathrm{~m})^{0.25}(\mathrm{~m} / \mathrm{s})^{0.5}$ & $\sim 1.0 \times 10^{-6} \mathrm{~m} \cdot \mathrm{s}^{-2}$ (gal.) & Grav. Accl. Const. \\
\hline 9) & $m_{p} / \rho_{p}^{0.25}=1.318 \times 10^{-15}$ & $1.352 \times 10^{-15}(\mathrm{~N} \mathrm{~m})^{0.75}$ & $2.068 \times 10^{-15} \mathrm{~Wb}$ & Mag. flux density \\
\hline 10) & $F_{w} / \tau_{w}^{2}=9.772 \times 10^{-24}$ & $9.676 \times 10^{-24} \mathrm{~N} \mathrm{~m}(\mathrm{rad} / \mathrm{s})^{2}$ & $9.285 \times 10^{-24} \mathrm{~J} \cdot \mathrm{T}^{-1}$ & Electron. mag. momt. \\
\hline 11) & $r_{w} / \sigma_{w}^{0.25}=7.482 \times 10^{-7}$ & $7.474 \times 10^{-7}(\mathrm{~N} \mathrm{~m})^{0.25}(\mathrm{~m} / \mathrm{s})^{0.25}$ & $12.566 \times 10^{-7} \mathrm{~N} \cdot \mathrm{A}^{-2}$ & Mag. perm. const. \\
\hline 12) & $F_{w} / \sigma_{w}^{0.5}=1.778 \times 10^{-12}$ & $1.755 \times 10^{-12}(\mathrm{~N} \mathrm{~m})^{0.5} \mathrm{~m} / \mathrm{s}^{-1}$ & $8.854 \times 10^{-12} \mathrm{~F} \cdot \mathrm{m}^{-1}$ & Electric. perm. const. \\
\hline
\end{tabular}

Table 2. Rotational properties of the isolated atom.

\begin{tabular}{|c|c|c|c|c|c|c|}
\hline Property & $\mathrm{E}$ & $\mathrm{H}$ & $\mathrm{Fe}$ & $\mathrm{Br}$ & $\mathrm{Ba}$ & $\mathrm{U}$ \\
\hline $\mathcal{V}_{w} / \mathrm{s}^{-1}$ & 1.00 & 2048.00 & $2.10 \times 10^{6}$ & $3.93 \times 10^{6}$ & $1.34 \times 10^{8}$ & $4.83 \times 10^{9}$ \\
\hline $\mathcal{\vartheta}_{p} / \mathrm{s}^{-1}$ & 2.00 & 3072.00 & $1.15 \times 10^{5}$ & $1.64 \times 10^{5}$ & $2.81 \times 10^{5}$ & $4.87 \times 10^{5}$ \\
\hline$\omega_{W} / \mathrm{rad} \mathrm{s}^{-1}$ & 6.28 & $1.29 \times 10^{4}$ & $1.32 \times 10^{7}$ & $2.47 \times 10^{7}$ & $8.43 \times 10^{8}$ & $4.05 \times 10^{10}$ \\
\hline$\omega_{p} / \mathrm{rad} \mathrm{s}^{-1}$ & 12.78 & $2.62 \times 10^{4}$ & $7.31 \times 10^{5}$ & $1.05 \times 10^{6}$ & $1.80 \times 10^{6}$ & $3.12 \times 10^{6}$ \\
\hline$r_{w} / \mathrm{m}$ & $1.5 \times 10^{8}$ & $7.32 \times 10^{4}$ & 71.48 & 38.10 & 1.10 & $3.10 \times 10^{-2}$ \\
\hline$r_{p} / \mathrm{m}$ & $9.1 \times 10^{-15}$ & $4.46 \times 10^{-18}$ & $1.60 \times 10^{-19}$ & $1.12 \times 10^{-19}$ & $6.49 \times 10^{-20}$ & $3.7510^{-20}$ \\
\hline$m_{w} / \mathrm{kg}$ atom $^{-1}$ & $7.37 \times 10^{-51}$ & $1.51 \times 10^{-47}$ & $1.54 \times 10^{-41}$ & $2.90 \times 10^{-41}$ & $9.88 \times 10^{-40}$ & $3.56 \times 10^{-38}$ \\
\hline$m_{p} / \mathrm{kg} \mathrm{u}^{-1}$ & $4.88 \times 10^{-7}$ & $1.00 \times 10^{-3}$ & 56.00 & 80.00 & 137.34 & 238.00 \\
\hline$\rho_{w} / \mathrm{kgm}^{-3}$ & $5.23 \times 10^{-76}$ & $9.19 \times 10^{-63}$ & $1.01 \times 10^{-50}$ & $1.25 \times 10^{-49}$ & $1.70 \times 10^{-43}$ & $2.85 \times 10^{-37}$ \\
\hline$\rho_{p} / \mathrm{kgm}^{-3}$ & $3.06 \times 10^{35}$ & $5.39 \times 10^{48}$ & $3.27 \times 10^{54}$ & $1.37 \times 10^{55}$ & $1.20 \times 10^{56}$ & $1.08 \times 10^{57}$ \\
\hline$F_{w} / \mathrm{N}$ & $4.36 \times 10^{-41}$ & $1.83 \times 10^{-34}$ & $1.92 \times 10^{-28}$ & $6.75 \times 10^{-28}$ & $7.86 \times 10^{-25}$ & $1.02 \times 10^{-21}$ \\
\hline$F_{p} / \mathrm{N}$ & $1.46 \times 10^{-18}$ & $6.11 \times 10^{-12}$ & $4.77 \times 10^{-9}$ & $9.75 \times 10^{-9}$ & $2.88 \times 10^{-8}$ & $8.6610^{-8}$ \\
\hline$\epsilon_{w} / \mathrm{Pa}$ & $2.91 \times 10^{-49}$ & $2.50 \times 10^{-35}$ & $2.68 \times 10^{-30}$ & $1.76 \times 10^{-29}$ & $7.04 \times 10^{-25}$ & $3.28 \times 10^{-20}$ \\
\hline$\epsilon_{p} / \mathrm{Pa}$ & $1.60 \times 10^{-4}$ & $1.37 \times 10^{6}$ & $2.98 \times 10^{10}$ & $8.74 \times 10^{10}$ & $4.44 \times 10^{11}$ & $2.31 \times 10^{12}$ \\
\hline$\sigma_{w} / \mathrm{Pa}$ & $6.18 \times 10^{-58}$ & $1.09 \times 10^{-44}$ & $1.20 \times 10^{-32}$ & $1.48 \times 10^{-31}$ & $2.00 \times 10^{-25}$ & $3.37 \times 10^{-19}$ \\
\hline$\sigma_{p} / \mathrm{Pa}$ & $5.56 \times 10^{9}$ & $9.79 \times 10^{22}$ & $5.95 \times 10^{28}$ & $2.49 \times 10^{29}$ & $2.18 \times 10^{30}$ & $1.96 \times 10^{31}$ \\
\hline$\tau_{w} / \%$ & $2.12 \times 10^{-11}$ & $4.35 \times 10^{-8}$ & $4.45 \times 10^{-5}$ & $8.35 \times 10^{-5}$ & $2.85 \times 10^{-3}$ & 0.10 \\
\hline$\tau_{p} / \%$ & $3.49 \times 10^{11}$ & $7.14 \times 10^{14}$ & $1.99 \times 10^{16}$ & $2.85 \times 10^{16}$ & $4.90 \times 10^{16}$ & $8.50 \times 10^{16}$ \\
\hline
\end{tabular}


Table 3. Variation of atomic wave/particle radii ratio of the chemical element.

\begin{tabular}{ccccccccc}
\hline Element & e-He & Li-B & O-Na & Mg-K & Ti-Zr & Nb-Pr & Nd-Fr & Ra-Am \\
\hline $\log \left(r_{w} / r_{p}\right)$ & 22.2 & $22.5-22.2$ & 22.0 & $21.8-21.4$ & $20.9-20.0$ & $19.9-19.0$ & $18.9-18.5$ & $18.2-17.8$ \\
\hline
\end{tabular}

Table 4. Some observational effects of intrinsic atomic strain.

\begin{tabular}{|c|c|c|c|c|c|c|c|c|}
\hline \multicolumn{9}{|c|}{ Boson Field } \\
\hline Correl. & $\mathcal{\vartheta}_{w} / \tau_{w}$ & $m_{w} / \tau_{w}$ & $r_{W} \tau_{W}$ & $\rho_{w} / \tau_{w}^{4}$ & $\omega_{w} / \tau_{w}$ & $F_{w} / \tau_{w}^{2}$ & $\epsilon_{w} / \tau_{w}^{3}$ & $\sigma_{w} / \tau_{w}^{4}$ \\
\hline Coeff. & $4.7 \times 10^{8}$ & $3.55 \times 10^{42}$ & 0.3184 & $2.57 \times 10^{-41}$ & $2.96 \times 10^{9}$ & $9.77 \times 10^{-24}$ & $3.09 \times 10^{-23}$ & $3.09 \times 10^{-23}$ \\
\hline Effect & Radiation & polarizability & $1 / \pi$ & Polarizability & Magnetic & $\mu_{e}$ & $\mu_{\mathrm{au}}$ & $\mu_{\mathrm{au}}$ \\
\hline \multicolumn{9}{|c|}{ Fermion Field } \\
\hline Correl. & $\vartheta_{p} / \tau_{p}$ & $m_{p} / \tau_{p}$ & $r_{p} \tau_{p}$ & $\rho_{p} / \tau_{p}^{4}$ & $\omega_{p} / \tau_{p}$ & $F_{p} / \tau_{p}^{2}$ & $\epsilon_{p} / \tau_{p}^{3}$ & $\sigma_{p} / \tau_{p}^{4}$ \\
\hline Coeff. & $5.89 \times 10^{-14}$ & $2.82 \times 10^{-20}$ & 0.3184 & $2.09 \times 10^{-20}$ & $7.31 \times 10^{-14}$ & $1.2 \times 10^{-45}$ & $3.8 \times 10^{-45}$ & $3.8 \times 10^{-45}$ \\
\hline Effect & Radiation & Charge & $1 / \pi$ & Charge & Radiation & Torque & Torque & Torque \\
\hline
\end{tabular}

Table 5. The particulate atom's internal pressure $(\sigma / \mathrm{Pa})$ in $U_{p}^{o}$ and $U_{p}^{\prime}$.

\begin{tabular}{ccccccc}
\hline Element & $\mathrm{e}$ & $\mathrm{H}$ & $\mathrm{Fe}$ & $\mathrm{Br}$ & $\mathrm{Ba}$ & $\mathrm{U}$ \\
\hline$\left(U_{p}^{o}\right) \sigma / \mathrm{Pa}$ & $5.56 \times 10^{9}$ & $9.79 \times 10^{22}$ & $5.95 \times 10^{28}$ & $2.49 \times 10^{29}$ & $2.17 \times 10^{30}$ & $1.97 \times 10^{31}$ \\
$\left(U_{p}^{\prime}\right) \sigma / \mathrm{Pa}$ & $3.48 \times 10^{8}$ & $6.12 \times 10^{21}$ & $5.37 \times 10^{33}$ & $7.01 \times 10^{34}$ & $1.12 \times 10^{43}$ & $1.89 \times 10^{47}$ \\
\hline
\end{tabular}

Table 1 reveals as follows:

- Torque $\Gamma$ is generated within the isolated quantum envelope by self-interaction of the field dynamics, the causality includes several SHM parameters of the harmonic oscillator; notably, the fermionic stress-radius correlation $\sigma_{p} r_{p}^{4}=\Gamma_{p}=3.867 \times 10^{-47} \mathrm{~kg} \cdot \mathrm{m}^{3} \cdot \mathrm{s}^{-2}$ identifies with the primitive causality of particulate matter's scale-free intrinsic rotation, Obande [20].

- The parameter seems to differentiate radially in a convergent series within the quantum envelope, for the vacuum field it would account for effects associated with "spooky action-at-a-distance" observed in, say, Newtonian gravitation, it might also be responsible for atmospheric electric potential gradient, Macken ([32] [46]), Emiliani [47].

- Two classes of torque are distinguishable, a "simple" torque field defines with the familiar unit $\mathrm{N} \mathrm{m}$ as in Table 1 Equations (1), (2), (5), (7) and (9), the unit modifies with exponents that vary from 0.25 to 1.0. A "complex" torque field is one in which the simple torque is itself in perpetual tangential and/or radial motion as in Equations (3), (6), (8), (10) to (12); notably, complex torques are uniquely bosonic features, with the exception of (3) they motivate perpetual translational (actually, tangential) motion of bodies through free space and orbital motion within the atom. Observe the tangential velocities $\left(\mathrm{m} \cdot \mathrm{s}^{-1}\right), \quad v_{o}^{0.5}$, Equation (8); $v_{o}^{0.25}$, Equation (11) and $v_{o}$, Equation (12) where $v_{o}=\pi c_{o}=9.418 \times 10^{8} \mathrm{~m} \cdot \mathrm{s}^{-1}$, Obande [48], the units present the vacuum field with tremendous electrical (permittivity) potential. 
- The interactions described in (1) and (2) have been presented in reasonable detail, Obande [20], theory refutes the assignment of atomic unit of charge to the constant $1.6022 \times 10^{-19}$; it is a torque field, more in tune with angular momentum vector, i.e., electric potential than the scalar, electrostatic charge quantum, Obande [20].

- Attention is drawn to Equation (3). In terms of sheer design perfection, it reveals, arguably, the most intensive compressive force field in all of nature; it is a hydrostatic bosonic gravitational force field that compresses with a "bulk" speed of $(2.99792458 \times \pi)^{-2.3}\left(\mathrm{~m} \cdot \mathrm{s}^{-1}\right)^{-2.3}$ bolstered with a matching angular shear stress. We have argued elsewhere, Obande [20], that on atomic scale, it motivates natural radioactivity and on cosmic scale, it motivates stellar explosion or supernova.

\subsection{Rotational Properties of the Isolated Atom}

It refers to the non-bonded atom subjected to none other than its own field, an ideal theoretical entity absolutely impossible to isolate or shield from the vacuum field. Thus, the properties listed in Table 2 refer to theoretical values, yet, some, e.g., theoretical electron radius $r_{e(p)}=9.1 \times 10^{-15} \mathrm{~m}$, come quite close to the classical value $=2.82 \times 10^{-15} \mathrm{~m}$; we highlight some physical characteristics of the quantum harmonic oscillator:

- Oscillation frequency $\vartheta$ and angular speed $\omega$ : Values of $\vartheta$ and $\omega$ vary by nine and ten orders of magnitude respectively from start to the end of the chemical periodicity, notably, both quantities increase with mass number in sequential geometric series in which the segment coincides with the chemical period.

- Atomic Radius $r$. Fundamentally, isotropic spatial dimension quantifies in mass/density quotient, $r=0.62038(\mathrm{~m} / \rho)^{0.333}$; for a harmonic oscillator the value also retrieves with $r=\lambda / 2$, or centripetal force/elastic modulus quotient, $r=F / \epsilon$. In contrast with the bonded atom, radius of the isolated atom decreases exponentially with mass number and also in segments that coincide with the chemical periods, see Figure 1; its value at the beginning and end of the periodicity differs by ten and five orders of magnitude for the atomic wave and particulate forms respectively. A plot of values of $r_{w}$ vs. $r_{p}$ in Figure 2 reproduces a familiar bimodal gradient commonly encountered in classical analysis of physical properties of the atom, Obande ([27] [43]). From e to H, comprising 23 inaccessible elements, we have a perfect linear correlation for which $\log \left(r_{w} / r_{p}\right)=L=22.2$; from He onwards, the value decreases logarithmically in measured periodic sequences to give $L_{\mathrm{He}}=22.2$ and $L_{\mathrm{Am}}=17.8$, see Table 3. In some cases the value is held constant over an interval, e.g., e to $\mathrm{He}, \mathrm{O}$ to $\mathrm{Na}$ and $\mathrm{Ni}$ to $\mathrm{Se}$, in others only one decimal place separates any two neighboring elements such as in the range Nd to Fr. These details necessitate with hope to probe causality of physical (gaseous, liquid or solid) states of the elements at standard conditions but available information proved inadequate hence we make only the following generalizations: 1) with a few 
exceptions, most elements registering with $r_{w}=1.622 \times 10^{22} r_{p}$, are either invisible or gaseous; 2 ) the elements $\mathrm{H}$ to $\mathrm{He}$ (five in number, Obande [42] constitute a visible-invisible condensed matter transition, they share common spacetime characteristics with both visible and invisible phases of reality, Obande [43]; 3) with the exception of a few gases and liquids, most elements with $\log \left(r_{w} / r_{p}\right)=21.8$ to 18.5 are solids, however, the element becomes spontaneously radioactive at $\left.\log \left(r_{w} / r_{p}\right) \leq 18.5 ; 4\right)$ electron wave or particulate form has the largest atomic radius of all elements hence, in the vacuum field and in bonded systems, the electron field encloses all other elements' fields, this position can be checked against existing x-ray diffraction rings of compounds containing $\mathrm{H}$ atoms, e.g., benzene, where the $\mathrm{H}$ ring would be found to enclose C rings, Levine ([49], p. 877); visible reality actually resides inside the electron waveform!, $r_{e(w)}=1.499 \times 10^{8} \mathrm{~m}$.

- Rest mass: It defines in the expression amu/ev $=k=\tau /(\omega / r)^{1 / 2}$ where $k=$ $1.037528 \times 10^{-5}$ and $9.31494 \times 10^{5}\left(\mathrm{rad} \mathrm{s}^{-1} \mathrm{~m}^{-1}\right)^{-1 / 2}$ are amu/MeV of the bosonic and fermionic quantum fields respectively, observe that the value $9.31494 \times 10^{5}$ corresponds to empirical $\mathrm{amu}=931.4 \mathrm{MeV}$, observe also that the unit reveals the parametric definition of the $\mathrm{eV}$. Interestingly, reduction of the element's strain to hydrogen's value also retrieves relative atomic mass, i.e., $\tau_{E} / \tau_{H}=m_{r} \mathrm{u}^{-1}$, Obande [42].

- Density $\rho$ : Elemental bosonic density varies from electron's $\rho_{e(w)}=5.23 \times 10^{-76} \mathrm{~kg} \cdot \mathrm{m}^{-3}$ to uranium's $\rho_{U(w)}=2.85 \times 10^{-37} \mathrm{~kg} \cdot \mathrm{m}^{-3}$, while corresponding fermionic densities are $\rho_{e(p)}=3.06 \times 10^{35}$ to $\rho_{U(p)}=1.08 \times 10^{57} \mathrm{~kg} \cdot \mathrm{m}^{-3}$. It reveals an invisible condensed matter field over $10^{100}$ denser than the vacuum field thus, visible reality floats in a pool of very dense invisible ("dark") particulate matter. Notably, bosonic densities of the chemical elements sum up to give vacuum material density $\rho_{\text {vac }}=2.6089 \times 10^{-39} \mathrm{~g} \cdot \mathrm{cm}^{-3}$ and the cosmological lambda $\Lambda=4.87 \times 10^{-66} \mathrm{~cm}^{-2}$, Obande [50].

- Centripetal force $F$ : As shown in Table 1, it motivates quite a number of effects: electrical, Equations (10), (12); magnetic, Equation (11); mechanical, Equation (2) and spatial dimension in both the boson and fermion fields. We attribute the bosonic field correlation coefficient $F_{w} / m_{w}^{2}=7.9433 \times 10^{59} \mathrm{~m} \cdot \mathrm{s}^{2} \cdot \mathrm{kg}^{-1}$ to the strong nuclear force SNF, it holds matter together on all scales from the atom to cosmos, Obande [20].

- Elastic (tensile) modulus $\epsilon$ : Vacuum material modulus varies across the chemical periodicity from e's $\epsilon_{w} / \mathrm{Pa}=2.91 \times 10^{-49}$ to $U=3.28 \times 10^{-20}$, corresponding values for the fermionic field are $\epsilon_{p}=1.60 \times 10^{-4}$ to $2.21 \times 10^{12}$ $\mathrm{Pa}$ for e to $\mathrm{U}$ respectively. Since the values refer to the isolated atom, the results reveal a highly elastic electromagnetic e-m vacuum spacetime fabric.

- Strain $\tau$. Intrinsic strain rate on the elemental bosonic quantum varies across the chemical periodicity from $2.12 \times 10^{-11} \%$ to $0.10 \%$ for electron to uranium, corresponding values for condensed matter are $3.49 \times 10^{11} \%$ to $8.6 \times 10^{16} \%$. The values follow from the quantitative expression $\tau=2 \vartheta / \pi c=\omega / \pi^{2} c$ where 
$\mathcal{\vartheta}, \omega$ and $c$ are oscillation frequency, angular speed and the transverse field respectively, for the vacuum $c_{o}=2.99792458 \times 10^{8}$ " $\mathrm{m} \cdot \mathrm{s}^{-1}$ " and for condensed matter $c^{o}=3.71535229 \times 10^{-14} " \mathrm{~m} \cdot \mathrm{s}^{-1}$ ". Strain correlates with a number of other physical properties to manifest electro-magnetism, mechanical properties and spatial dimensions, Obande [20], a small sample is presented in Table 4.

\subsection{Observational Effects of Intrinsic Rotation}

Recall that complex torques (Equations (3), (8), (10), (11) and (12), Table 1 are simple torques in perpetual motion in free space or in matter; some, e.g., (3), combine tangential and angular motions, others, e.g., (8), (11) and (12) execute sub- and super-luminal velocities, i.e., $v_{o}^{0.25}, v_{o}^{0.5}$ and $v_{o}$, where $v_{o}=\pi c_{o}$, yet others, e.g., (10) attribute to only angular speed. A detailed presentation of the subject would lengthen this report far beyond the intended scope; we highlight only some key observational effects.

\subsubsection{Metric Expansion of Space and Matter}

All natural spatial periodic quanta are ellipsoids, see, e.g., the "Static Sky", New Castle [51] and the galaxies in Galaxy [52]. The morphology provides an important clue to the profile of metric space expansion, there are only two straight (axial) lines in an ellipsoid-the major and minor axes; in cosmic envelopes these two directions are totally forbidden on account of the (galactic) nucleus; in condensed matter the nucleus is encased in a shell of fermionic matter but, on account of gravity, remains impassable. In nature, therefore, projectiles circumvent the nucleus and trace only geodesics (parabolas), see Physics Forum.org [16]. The "Static Sky" provides an excellent perspective, condensed matter fields are constrained within the vertical cylindrical elliptic envelope, it constrains expansion to within the toriod. A superluminal tangential velocity that traces a larger ellipsoid creates the impression of radial acceleration of space, Castelvicchi [53], Nielson et al. [54], Brax [55], Billings [56], it is motivated by the bosonic field coupling $\rho_{w} / \sigma_{w}=8.5114 \times 10^{-19},\left(\mathrm{~m} \mathrm{rad} \mathrm{s}^{-1}\right)^{-2}$; theoretical analysis yields the expansion rate $v_{o}=0.5 \pi c_{o}=4.709 \times 10^{8} \mathrm{~m} \cdot \mathrm{s}^{-1}$, notably, it is measurable as the vacuum characteristic ("atmospheric electrostatic charge") $8.5 \times 10^{-19}$ "C", Obande [20].

\subsubsection{Motions in Free Space and in Condensed Matter}

In free space the complex torque field motivates spontaneous translational motion, i.e., inertia, Lynden-Bell [57] of bodies including galaxies, stars, satellites, comets, et cetera; notably, these motions are not random events, each occurs within a well-defined trajectory fixed at formation of the body. Of particular interest in this class of motions is the seeming expansion of space broached above but belongs to a very rich subject that touches upon the details of birth, growth and death of matter. In condensed matter, bonding restricts the "primary" motion modes of Equations (3), (8), (10), (11) and (12) to within a limited radius 
resulting in a "secondary" mode that comprises mostly rotation and vibration about fixed axes. The secondary mode gives rise to vital observational effects: spin identifies, of course, with the primitive torque fields quantitatively expressed in $\Gamma_{a u}$ and $\Gamma_{n u}$, Equation (4); orbital motion or revolution identifies with angular motion, see Equation (10), and recession attributes to a coupling having only rectilinear dimension as in Equations (8), (11), (12). Of course, the rectilinear dimension refers to tangential motion which, as noted above, creates the illusion of radial expansion. In reality it refers to a process that gradually transforms a given elliptical envelope to a larger one until the envelope disintegrates and disappears spewing its content into vacuum space as asteroid, comet, other trans-stellar/galactic voyager which eventually also disintegrates and disappears into the void. The process is the universal scale-free death process of all matter, atomic, elemental, stellar, galactic, chemical, geological and biological bodies, Obande [27].

Observe that the mobile torque field informs: 1) Newton's second law of motion where it accounts for sundry perpetual motion including: axial spin, orbital motion, and recession from the center e.g. moon from earth, BBC.com [58]; bulk expansion of cosmological bodies, e.g., earth, Diaz [59], sun, Appell [60] and expansion of the galaxy, Sciama, [61], Wall [62]. 2) Random Thermal (Brownian) Motion which, of course, is a condensed-phase internal motion limited by chemical bond to localized translational, rotational, vibrational, rocking and twisting modes. Interestingly, energies of these secondary modes quantize alongside the primary modes; as is well known, it enables applications in a variety of high-precision analytical devices, see, e.g., Levine (1988). 3) Kinetic Molecular Theory KT; Equations (8), (11) and (12) give the free-space tangential velocities $\left(\mathrm{v} / \mathrm{m} \cdot \mathrm{s}^{-1}\right): \pi c_{o}^{0.5}=3.069 \times 10^{4} ; \pi c_{o}^{0.25}=175.183$, and $\pi c_{o}=9.418 \times 10^{8}$. It implicates moving torque fields in the familiar effects associated with random (thermal) motion whose observational root-mean-square velocity $v_{r m s}=(3 R T / M)^{0.5}$. Substitution of electron molar and atomic mass values, $m_{e 2(p)} / \mathrm{kg} \cdot \mathrm{u}^{-1}=9.766 \times 10^{-7}$ and $m_{e(w)} / \mathrm{kg} \cdot$ atom $^{-1}=7.373 \times 10^{-51}$, yields $v_{r m s} / \mathrm{m} \cdot \mathrm{s}^{-1}=8.724 \times 10^{4}$ and $1.004 \times 10^{27}$ respectively. The molar value $8.724 \times$ $10^{4} \mathrm{~m} \cdot \mathrm{s}^{-1}$ tallies with $\pi c_{o}^{0.5}$ and speaks well in favor of consistency of both KT and the present classical mechanics CM approach; however, bosonic electron's $v_{r m s}=1.004 \times 10^{27} \mathrm{~m} \cdot \mathrm{s}^{-1}$ presents an entirely new speed limit scenario. KT is well established, it serves here to cross-check the values obtained with the CM approach. The indication here of existence in nature of velocity on the order of $10^{27} \mathrm{~m} \cdot \mathrm{s}^{-1}$ comes with tremendous implications specifically for on-going neutrino research but, the subject must await further investigation. The analysis clearly indicates that much of chemical kinetics and thermodynamics, particularly the concepts of enthalpy, entropy and thermodynamic temperature scale, easily trace to physics of the mobile torque field.

\subsubsection{Internal Pressure $\sigma$ of the Quantum Envelope}

Atomic stress (internal pressure) evaluates with $\sigma=F / \pi r^{2}=8 \pi m \vartheta^{3} / c$, Obande 
[48]. The value varies across the chemical periodicity from bosonic electron's $6.18 \times 10^{-58}$ to uranium's $10^{-19} \mathrm{~Pa}$, corresponding values for particulate e to $\mathrm{U}$ are $5.56 \times 10^{9}$ to $1.96 \times 10^{31} \mathrm{~Pa}$. In other words, the fermionic energy packet is some fifty orders of magnitude more internally pressurized than its bosonic conjugate. Burkert et al. [63] recently reported the value $\sigma_{\text {proton }} \sim 10^{35} \mathrm{~Pa}$; theoretical analysis gives $\sigma_{\text {proton }} / \mathrm{Pa}=9.79 \times 10^{22}$ and $6.12 \times 10^{21}$ for the visible proton and its invisible (mass generation) analogue respectively. Correct situation of the empirical and theoretical values requires unambiguous identification of the experimental proton's phase, Obande [43], i.e., its candidature among the three particle generations. We present in Table $5 \sigma$ values of some elements in our visible $U_{p}^{o}$ and in its invisible analogue $U_{p}^{\prime}$; clearly, $\mathrm{H}^{+}$does not register with $\sigma_{p} \sim 10^{35}$ in any ref. frame. The theoretical analysis therefore suggests a possibility that either the experimental set up over-estimates $\sigma_{\text {proton }}$ or, a rogue non-visible particulate element other than the proton is involved. We must, however, observe that in the course of this project we have uncovered significant divergences between theoretical and empirical atomic property values, e.g., a whopping twenty-order magnitude exists between electron empirical rest mass $9.1 \times 10^{-31}$ and theoretical value $7.37 \times 10^{-51} \mathrm{~kg} \cdot$ atom $^{-1}$, Obande [42]. However, there is no doubt that Burkert et al. [63]'s result makes an indispensable contribution to the position that the condensed matter energy packet is a highly pressurized vessel, see Zhou [64].

\section{Summary and Conclusions}

- Internal stress of a periodic quantum field correlates with the energy packet's radius to generate the intrinsic torque $\Gamma$ that motivates spontaneous rotation of matter, its atomic and natural units are electron's bosonic $\Gamma_{a u(w)}=\sigma_{w} r_{w}^{4}=3.162 \times 10^{-25} \mathrm{~kg} \cdot \mathrm{m}^{3} \cdot \mathrm{s}^{-2}$ and fermionic $\Gamma_{a u(p)}=\sigma_{p} r_{p}^{4}=3.867 \times 10^{-47} \mathrm{~kg} \cdot \mathrm{m}^{3} \cdot \mathrm{s}^{-2}$.

- The evidence suggests that the field parameter differentiates radially in a convergent infinite series within the envelope to produce effects attributed to "spooky-action-at-a-distance" such as observed in Newtonian gravitation and in spatial electric potential gradient.

- An earlier report was cited to inform that in addition to stress and radius, several other field parameters correlate to generate torque; for instance, the all-too-familiar fundamental constant $1.6022 \times 10^{-19}$ attributes to the correlation coefficient of three different parametric couplings: $\rho_{p} / \tau_{p}^{4} ; F_{p} / \vartheta_{p}^{2}$ and $\rho_{w} / \vartheta_{w}^{4}$ where $\rho$, $\tau$, and $\vartheta$ are flux density, strain and frequency and indices $\mathrm{p}$ and $\mathrm{w}$ denote fermionic and bosonic fields respectively.

- As a result of intrinsic rotation, all bodies possess characteristic harmonic motion parameters including frequency, radius, mass, density, centripetal force, modulus, stress and strain. Since aggregate waveforms of the chemical elements constitute the vacuum field, Obande [44], the vacuum is actually an ideal elastic body defined with SHM properties of elemental waveforms; 
notably, the vacuum-value (amplitude) of a given property is not an average but sum total of values of elements of the chemical periodicity; e.g., vacuum density sums up to give the cosmological lambda, Obande [50].

- The correlations describe conic sections, it accounts for ellipsoidal morphology of cosmic objects and rules out any notion of linear trajectory in nature, all seeming linear motions are tangential to larger geodesics; in other words, metric space cannot expand radially, it is an angular phenomenon.

- As found in previous cases, theoretical results in this series call for caution in making deductions from particle physics experiments; we find, consistently, results which suggest that experimental energy regimes often diverge markedly from theoretical values. In the case in point, Burkert et al.'s recently reported proton internal pressure $\sigma_{\mathrm{H}^{+}} \sim 10^{35} \mathrm{~Pa}$ differs significantly from the theoretical value of each of the proton's three particle generations, specifically, $\sigma_{\mathrm{H}^{+}} / \mathrm{Pa}=9.79 \times 10^{22}$ and $6.11 \times 10^{21}$ in $U_{p}^{o}$, and $U_{p}^{*} / U_{p}^{\prime}$ respectively. Theoretical analysis reveals that $\sigma$ value in the neighborhood of $10^{35}$ registers only for invisible (“dark-matter") trans-bromium elements, not earlier.

- Restricted rotation in condensed matter creates all manner of modes of motion including, random thermal (Brownian), vibtrational, bending, rocking, twisting, et cetera. These modes are quantized in line with the causal harmonics, they manifest the spectrum of effects quantitatively associated with thermodynamics, kinetics and, in particular, kinetic-molecular theory. Most notably, the present CM approach leads to evaluation of the root-meansquare velocity $v_{r m s}=(3 R T / M)^{0.5}$ for which electron waveform's $m_{e(w)}=7.3725 \times 10^{-51} \mathrm{~kg} /$ atom, gives $v_{r m s(e w)}=1.004 \times 10^{27} \mathrm{~m} \cdot \mathrm{s}^{-1}$; this result, suggesting existence of an imponderable hyper-luminal velocity, comes with important implications for on-going neutrino research, it is, however, set aside for further investigation.

The investigation has succeeded in explicitly accounting for the "mystery" of rotation such as proton spin, Moscowitz [65]; if taken with our earlier report on morphology of cosmological bodies, the present results point to a link between rotation and figure of celestial bodies St Katlin [66]. Notably, "gravitational accretion" is not in any way implicated in intrinsic rotation, Giuli [67]. It has become customary, in concluding a report of an investigation in this series, to call attention to the sheer power of the unassuming expression $h \vartheta=m c^{2}$; it, of course, equates energies of the composite wave and particulate forms of the atom and in effect quantifies the atom's essence and therein lies its analytical power. We do not think a simpler, yet more powerful, dual energy quantification is feasible, therefore, we submit the Planck-Einstein-de Broglie (PEB) mass equation the ultimate simplification of The Theory of Everything. In order to demonstrate its incredible simplicity and awesome analytical power, we have, quite deliberately, used the PEB to address areas considered intractable in the reigning physics paradigm, e.g., origin of the three-particle generations and identity of 
"dark" matter/energy, Obande [34]; elemental intrinsic atomic e-m resonance frequency, 9-value, Obande [44]; common causality of gravitation, electricity and magnetism, Obande [45]; atomic mass phenomenology, Obande [42]; cosmological constant phenomenology, Obande [50]; the photon's identity, Obande [48]; phenomenology of the fundamental physical constants, Obande [20] and herein, origin of intrinsic rotation of matter. We have, in each case, submitted compelling positions that as yet await independent assessment. The goal is to assemble what would eventually become foundational materials of an all-embracing classical atomic theory with which an observational theory of nature is realizable. We think, even without going further, we have already assembled sufficient materials for development of an observational theory of nature.

\section{Conflicts of Interest}

The author declares no conflicts of interest regarding the publication of this paper.

\section{References}

[1] Ellis, G. and Silk, J. (2014) Scientific Method: Defend the Integrity of Physics. Nature, 516, 321-323. https://doi.org/10.1038/516321a

[2] Beth, R.A. (1936) Mechanical Detection and Measurement of the Angular Momentum of Light. Physical Review, 50, 115-125. https://doi.org/10.1103/PhysRev.50.115

[3] Leach, J., Courtial, J., Sheldon, K., et al. (2004) Interferometer Methods to Measure Orbital and Spin or the Total Angular Momentum of a Single Photon. Physical Review Letters, 92, Article ID: 013601. https://doi.org/10.1103/PhysRevLett.92.013601

[4] Jaffe, R.L. (1995) Where Does the Proton Get Its Rotation. Physics Today, 48, 24-30.

[5] Peterson, I. (1997) "Proton-Go-Round"-Where Does the Proton Get Its Spin? Science News, 152, 158-159. https://doi.org/10.2307/3981051

[6] Raghuprasad, P.K. (2013) Planetary Spin-Orbit Attributes in the Solar System and Their Wider Implications. Physics Essays, 26, 331-338.

https://doi.org/10.4006/0836-1398-26.2.331

[7] Tsati, A., Lyubenova, M., et al. (2017) CALIFA Reveals Prolate Rotation in Massive Early-Type Galaxies: A Polar Galaxy Merger Origin? Astronomy \& Astrophysics, 606, A62. https://doi.org/10.1051/0004-6361/201630218

[8] McClintock, J.E., Shafee, R. and Narayan, R. (2006) The Spin of the Near Black Hole GRS $1915+105$. The Astrophysical Journal, 652, 518-539.

https://doi.org/10.1086/508457 https://arxiv.org/abs/astro-ph/0606076

[9] Imanishi, M., Nakanishi, K., Izumi, T. and Wada, K. (2018) Alma Reveals an Inhomogeneous Compact Rotating Dense Molecular Torus at NGC 1068 Nucleus. The Astrophysical Journal Letters, 853, L25. https://doi.org/10.3847/2041-8213/aaa8df

[10] Williams, J.G. and Mark, M.B. (1997) Is the Electron a Photon with Toriodal Topology? Annales de la Fondation Louis de Broglie, 22, 133.

[11] Shea, S.B. (2017) How Did the Proton Get Its Spin? https://physics.org/news/2017-03-proton.html

[12] Spagna, G. (2018) Why and How Do Planets Rotate? https://www.scientificamerican.com/article/why-and-how-do-planets-ro 
[13] Palacios, A. (2013) Influence of Rotation on Stellar Evolution. https://arxiv.org/abs/1307.1316

[14] Benesch, C. (2013) Why Does Every Astronomical Object Rotate? Where Has the Angular Momentum Come from?

https://www.quora.com/Why-does-every-astronomical-object-rotate-where-does-th e-angular-momentum-come-from-infact-how-do-neutron-stars-possess-such-

[15] Crawford, C. (2013) Rotation in Space. Atronomy Lectures, Gresham College, Cambridge. https://www.gresham.ac.uk/lectures-and-events/rotation-in-space

[16] Young, A. (2018) How Does Space Being Curved Make Gravity Pull Us Down? Physics Discussion Forum.org. https://www.quora.com/How-does-space-being-curved-make-gravity-pull-us-down

[17] Dirac, P.A.M. (1928) The Quantum Theory of the Electron. Proceedings of the Royal Society $A, 117,610$.

Dirac, P.A.M. (1939) The Relation between Mathematics and Physics. Proceedings of the Royal Society, 59, 122-129. https://doi.org/10.1017/S0370164600012207

[18] Born, M. (1935) The Mysterious Number. https://www.oldias.ac.in/j_archive/proca/2/top.html

[19] Golubev, S.N. (2014) The Emergence and Existence of Life in the World of Elements and the Physical Vacuum. Achievements in the Life Sciences, 8, 1-9. https://doi.org/10.1016/j.als.2014.11.001

[20] Obande, O.P. (2017) On the Fundamental Physical Constants: I. Phenomenology. Applied Physics Research, 9, 42. https://doi.org/10.5539/apr.v9n5p42

[21] Hossenfelder, S. (2018) Lost in Math: How Beauty Led Physics Astray. Basic Books, New York.

[22] Wilczek, F. (2018) Comment on: Has Elegance Betrayed Physics? Physics Today, 71, 57. https://doi.org/10.1063/PT.3.4022

[23] Rugh, S.E. and Zinkernagel, H. (2001) The Qunantum Vacuum and the Cosmological Constant Problem. https://arxiv.org/hep-th/0012253

[24] Rees, M.J. (1985) Galaxies and Their Nuclei. The Bakerian Lecture, 1982. Proceedings of the Royal Society of London. Series A, 400, 183.

https://doi.org/10.1098/rspa.1985.0076

https://rspa.royalsocietypublishing.org

[25] Cross, D.J. (2015) The Physical Origin of Torque and the Rotational Second Law. American Journal of Physics, 83, 121-125. https://doi.org/10.1119/1.4896574

[26] Gorbatyy, I.N. and Tarsov, A.A. (2017) Another Comment on: “The Physical Origin of Torque and of the Second Law". American Journal of Physics, 85, 315-317. https://doi.org/10.1119/1.4975383

[27] Obande, O.P. (2017b) On the Fundamental Physical Constants: II. Field Coupling Geometry. Applied Physics Research, 9, 62. https://doi.org/10.5539/apr.v9n5p62

[28] SCIAM (2013) Physicists Debate Whether the World Is Made of Particles or Fields-Or Something Else Entirely.

https://www.scientificamerican.com/article/physicists-debate-whether-the-world-m ade-of-particles-fields-or-something-else/

[29] Kuhlman, M. (2013) What Is Real? https://foglianuova.files.wodpress.com/2013/10/whatisreal.pdf

[30] Descartes, R. (1644) Prinzipien Philisophie. Teil 3, van der Sichtharen Welt, Absnitt. 23.

[31] Madelung, E. (1927) Quantum Theory in Hydrodynamic Form. Zeitschrift für Phy- 
sik, 40, 322-326. https://doi.org/10.1007/BF01400372

[32] Macken, J.A. (2011) The Universe Is Only Space. https://onlyspacetime.com/

[33] Hobson, A. (2013) There Are No Particles, There Are Only Fields. American Journal of Physics, 81, 211-223. https://doi.org/10.1119/1.4789885

[34] Obande, O.P. (2013) Notes on Russellian Cosmogony-Part 1: Absolute Atomic Mass. International Journal of Engineering Science, 2, 68. https://wwww.theijes.com/papers/v2-i4/part.\%20(2)/M0242068077.pdf

[35] Consiglio, J. (2015) How and Why the Universe Is Natural. Applied Scientific Research, 7, 69. https://doi.org/10.5539/apr.v7n3p69

[36] Kirakosyan, G. (2015) Deduction of the Coupling Constant $(a \approx 1 / 137)$ as a Wave Perculiarity: Possible Laboratory Confirmation. Physics Essays, 28, 283-285. https://doi.org/10.4006/0836-1398-28.2.283

[37] Wilczek, F. (1998) The Persistence of the Ether. Physics Today, 52, 11-13. https://doi.org/10.1063/1.882562

[38] Colbeck, R. and Renner, R. (2012) Is a System's Wave Function in One-to-One Correspondence with Its Elements of Reality? Physical Review Letters, 108, Article ID: 150402. https://doi.org/10.1103/PhysRevLett.108.150402

[39] Laidlaw, A. (2017) Relativity and the Luminal Structure of Matter. Progress in Physics, 13, 35.

[40] Musser, G. (2018) What Is Spacetime? https://doi.org/10.1038/scientificamerican0618-55 https://www.scientificamerican.com/article/what-is-spacetime

[41] Good Elf (2016) Theory of Particles Based on Vortices. https://physicsdiscussionforum.org

[42] Obande, O.P. (2016) Atomic Mass: Origin, Units and Constants. Applied Physics Research, 8, 92. https://doi.org/10.5539/apr.v8n1p92

[43] Obande, O.P. (2018) A Field Concept of the Black Hole. Applied Physics Research, 10, 66-80. https://doi.org/10.5539/apr.v10n4p66

[44] Obande, O.P. (2015) Notes on Russellian Cosmogony. II. A Procedure for Theoretical Calculation of Relative Atomic Mass and Internal Energy, Physics Essays, 28, 78. https://doi.org/10.4006/0836-1398-28.1.78

[45] Obande, O.P. (2015) Classical Definitions of Gravitation, Electricity and Magnetism. Applied Physics Research, 7, 85. https://doi.org/10.5539/apr.v7n6p85

[46] Macken, J.A. (2015) Spacetime Based Foundation of Quantum Mechanics and General Relativity. In: Nascimento, M. and Maruani, J., Eds., Osvaldo Goscinski (1938-2013), Springer, Berlin, Vol. 29, 219-245. https://doi.org/10.1007/978-3-319-14397-2_13

[47] Emiliani, C. (1992) Planet Earth, Cosmology, Geology and Evolution of Life and Environment. Cambridge University Press, Cambridge, 601-604.

[48] Obande, O.P. (2016) On the Photon's Identity: Implications for Relativity and Cosmology. Applied Physics Research, 8, 10. https://doi.org/10.5539/apr.v8n5p10

[49] Levine, I.N. (1998) Physical Chemistry. 3rd Edition, McGraw-Hill, New York, 440-450.

[50] Obande, O.P. (2016b) A Classical Perspective of the Cosmological Constant. Physics Essays, 29, 228-231. https://doi.org/10.4006/0836-1398-29.2.228

[51] New Atlas (2016) Static Universe. https://newatlas.com/pan-sarrs-largest-digital-survey-visible-universe 
[52] Galaxy (2018) Wikipedia, the Free Encyclopedia. https://en.wikipedia.org/wiki/Galaxy

[53] Castelvecchi, D. (2016) Measurement of Universe's Expansion Rate Creates Cosmological Puzzle. Nature News \& Comments. https://doi.org/10.1038/nature.2016.19715

[54] Nielson, J.T., Guffanti, A. and Sakar, S. (2016) Marginal Evidence for Cosmic Acceleration from Type 1A Supernovae. Scientific Reports, 6, Article No. 35596. https://doi.org/10.1038/srep35596

[55] Brax, P. (2018) What Makes the Universe Accelerate? Reports on Progress in Physics, 81, Article ID: 016901. https://doi.org/10.1088/1361-6633/aa8e64

[56] Billings, L. (2018) Cosmic Conflict: Diverging Data on Universe's Expansion Polarizes Scientists.

https://www.scientificamerican.com/article/cosmic-conflict-diverging-data-on-univ erses-expansion-polarizes-scientists1/

[57] Lynden-Bell, D. (1967) On the Origins of Space-Time and Inertia. Monthly Notices of the Royal Astronomical Society, 135, 413-428.

https://doi.org/10.1093/mnras/135.4.413

[58] BBC.com (2011) Why the Moon Is Getting Further Away from the Earth. BBC News, July. https://www.bbc.com/news/science-environment-12311119

[59] Diaz, L. (2018) Africa Is Splitting into Two Continents. https://the-conversation.com/africa-is-splitting-into-two-here-is-why/94056

[60] Appell, D. (2008) The Sun Will Eventually Engulf Earth-Maybe. https://www.scientificamerican.com/article/the-sun-will-eventually-engulf-earth-m $\underline{\text { aybe }}$

[61] Sciama, D.W. (1969) Is the Galaxy Losing Mass on a Time Scale of a Billion Years? Nature, 224, 1263-1267. https://doi.org/10.1038/2241263a0

[62] Wall, M. (2018) The Milky Way Is Growing Faster than the Speed of Sound. https://www.space.com/40194-milky-way-galaxy-growing.html

[63] Burkert, V.D., Elouadrhini, L. and Girod, F.X. (2018) Pressure Distribution inside the Proton. Nature, 557, 396-399. https://doi.org/10.1038/s41586-018-0060-Z

[64] Zhou, M. (2003) A New Look at the Atomic Level Virial Stress: On ContinuumMolecular System Equivalence. Proceedings of the Royal Society of London. Series $A$, 459, 2347-2392. https://doi.org/10.1098/rspa.2003.1127

[65] Moskowitz, C. (2014) Proton Spin Mystery Gains a New Clue. Scientific American. https://www.scientificamerican.com/article/proton-spin-mystery-gains-a-new-clue1/

[66] St. Katlin, N. (1961) On the Rotation and Figure of Celestial Bodies. Astronomische Nachrichten, 286, 157. https://doi.org/10.1002/asna.19612860404

[67] Giuli, R.T. (1968) On the Rotation of the Earth Produced by Gravitational Accretion. Science, 8, 301-323. https://doi.org/10.1016/0019-1035(68)90082-1 